\title{
Perda de peso rápida em jovens competidores de Taekwondo
}

\author{
Rapid weight loss in Taekwondo young competitors
}

\section{Rossano Diniz' ${ }^{1}$, Larissa da Cruz Braga ${ }^{2}$, Fabrício Boscolo Del Vecchio ${ }^{3}$}

${ }_{1}^{1}$ Pedagogo, Especialista em Gestão da Educação, Supervisão e Orientação Escolar, Integrante do Grupo de Pesquisas em Treinamento Esportivo e Desempenho Físico, Escola Superior de Educação Física, Universidade Federal de Pelotas (UFPEL), Pelotas, RS; ${ }^{2}$ Nutricionista, Integrante do Grupo de Pesquisas em Treinamento Esportivo e Desempenho Físico - Escola Superior de Educação Física, UFPEL, Pelotas, RS; ${ }^{3}$ Professor de Educação Física, Líder do Grupo de Pesquisas em Treinamento Esportivo e Desempenho Físico - Escola Superior de Educação Física, UFPEL, Pelotas, RS, Grupo de Estudos e Pesquisas em Lutas, Artes Marciais e Modalidades Esportivas de Combate - Escola de Educação Física e Esporte, Universidade de São Paulo (USP), São Paulo, SP.

\section{RESUMO}

Objetivos: Verificar a frequência, as características e os métodos usados para a perda rápida de peso por lutadores de Taekwondo do estado do Rio Grande do Sul.

Métodos: Um estudo transversal recrutou atletas que preencheram os seguintes critérios de inclusão: a) ser lutador de Taekwondo, b) graduado em faixa preta e c) competidor no evento em que ocorreu a coleta dos dados. A coleta dos dados foi realizada durante a primeira etapa do Circuito Costa Doce - Seletiva para Seleção Gaúcha, em 2013. Os dados foram coletados com questionário validado sobre perda de peso em lutadores, composto por três seções, preenchido antes da pesagem.

Resultados: Dos 23 voluntários, 21 (91,3\%) relataram perder peso para competir. A maioria perdeu entre 1 e $3 \mathrm{~kg}$ no período anterior à competição, durante três a quatro dias, até duas vezes por ano, através dos seguintes métodos: aumento de atividade física, omissão de refeições e restrição da ingestão hídrica. A maioria dos homens perdeu de 1 a $2 \mathrm{~kg}$ e as mulheres entre 1 e $2,5 \mathrm{~kg}$. Os homens recuperaram até $2 \mathrm{~kg}$ após a pesagem, enquanto as mulheres chegaram a recuperar até $7 \mathrm{~kg}$. O mestre foi a pessoa que mais os influenciou.

Conclusões: Observou-se alta freqência da rápida redução de peso em atletas de Taekwondo. O profissional citado como maior influente na perda de peso foi o mestre. Os métodos mais utilizados foram o aumento de atividade física, diminuição na ingestão de alimentos e restrição hídrica. DESCRITORES: TAE KWON DO; ARTES MARCIAIS; PERDA DE PESO.

\section{ABSTRACT}

Aims: To assess frequency, characteristics and methods used for rapid weight loss by Taekwondo fighters in the state of Rio Grande do Sul Methods: A cross-sectional study recruited athletes who met the following inclusion criteria: a) to be a Taekwondo fighter, b) to have a black belt degree c) being competitor in the event in which data collection occurred. Data collection took place during the first stage of the Costa Doce Circuit - Selective to State Team in 2013. Data were collected using a validated questionnaire on weight loss in wrestlers, consisting of three sections, completed before weighing.

Results: Of the 23 volunteers, 21 (91.3\%) reported losing weight to compete. Most lost between 1 and $3 \mathrm{~kg}$ in the pre-competition period for three to four days, up to twice a year, by the following methods: increasing physical activity, skipping meals and restriction of fluid intake. Most men lost 1-2 kg and women between 1 and $2.5 \mathrm{~kg}$. Men recovered until $2 \mathrm{~kg}$ after weighing, while the women came to recover up to $7 \mathrm{~kg}$. The master was the person who most influenced them.

Conclusions: We observed a high frequency of fast weight reduction in Taekwondo athletes. Professional cited as most influential in weight loss was the Master. The methods used were increased physical activity, decreased intake of food and fluid restriction.

KEY WORDS: TAE KWON DO; MARTIAL ARTS; WEIGHT LOSS.

Recebido em outubro de 2013; aceito em março de 2014. 


\section{INTRODUÇÃO}

A perda de peso é fenômeno comum entre lutadores, ocorrendo de modo crônico, quando superior a duas semanas, ou agudo, se realizada no período pré-competitivo. Esse processo deriva da redução da massa corporal total, principalmente em decorrência de restrição da ingestão calórica e hídrica, emprego de substâncias termogênicas, aumento da quantidade de atividades físicas intensas e submissão a ambientes de alta temperatura, com ou sem o uso de agasalhos térmicos. ${ }^{1}$

Tal prática é empregada com vistas a campeonatos das diferentes modalidades esportivas de combate, dado que os atletas são alocados em categorias divididas de acordo com a massa corporal. ${ }^{2}$ Assim, a pesagem passa a ter relevância elevada nas competições, dado que os lutadores diminuem seu peso estimando lutar em categorias mais leves, com a intenção de enfrentarem adversários menores e mais fracos. ${ }^{3}$ Neste contexto, geralmente se perde, de forma aguda, uma média de 5 a $10 \%$ do peso habitual. ${ }^{4,2}$

Especificamente no Taekwondo (TKD), a World Taekwondo Federation (WTF) adota dois tipos diferentes de divisões de massas corporais em competições, uma para o Campeonato Mundial, com a divisão de oito categorias de peso, e outra para os Jogos Olímpicos, com quatro categorias. A primeira é a mais usada, porque esse evento ocorre de dois em dois anos e as seletivas nacionais e continentais são realizadas nesses mesmos moldes. Ainda quanto ao TKD, estudos demonstram que um grande número de lutadores tenta perder peso para lutar em categorias mais leves, com objetivo de obter melhor desempenho, ocasionando o "weight cycling", expressão usada para definir o efeito sanfona causado pela perda aguda de massa corporal e retorno rápido ao estado antigo logo após a pesagem. ${ }^{5}$ Já se observaram reações negativas nesse processo, as quais foram relatadas pelos atletas, como cansaço, diminuição do rendimento, aumento da tensão, redução do vigor, aumento da raiva, tontura e maior suscetibilidade a resfriados e infecções. ${ }^{6}$

Mesmo sabendo dos possíveis prejuízos fisiológicos em função da perda de peso aguda na fase pré-competitiva, competidores realizam tais procedimentos acreditando na recuperação através da ingestão de alimentos e líquidos entre o período pós-pesagem e o início das lutas. ${ }^{7}$ Porém, especialmente no TKD, tais práticas nem sempre conduzem a melhoras no desempenho em competições, dado que lutadores jovens que obtêm maior índice de perda de massa corporal não são os atletas medalhistas na maioria das categorias. $^{8}$

Considerando a escassez de estudos nacionais sobre perda de peso em atletas de TKD e a relevância da temática acerca dos prejuízos agudos e crônicos causados por essa prática, o presente estudo objetivou verificar a frequência da rápida redução de peso corporal nos últimos 12 meses antes da competição e os métodos usados para atingi-la, em um grupo de competidores de nível estadual do estado do Rio Grande do Sul, Brasil.

\section{MÉTODOS}

O estudo, do tipo transversal, envolveu lutadores de ambos os sexos que eram competidores de TKD no ano de 2012. Como critérios de inclusão, consideraram-se: 1) ser lutador de TKD, 2) ser graduado como faixa preta e 3) ser competidor no evento em que ocorreu a coleta dos dados. O projeto teve aprovação do Comitê de Ética em Pesquisa da Escola de Educação Física da Universidade Federal de Pelotas, sob número 003/2012.

A coleta dos dados ocorreu durante a etapa seletiva para composição da Seleção Gaúcha do ano de 2013, da Federação Gaúcha de Taekwondo (FGT), a qual autorizou a realização do estudo. Esse evento ocorre anualmente, com três etapas na região sul do Rio Grande do Sul e outras três na região metropolitana. Todos os participantes foram recrutados durante o campeonato da primeira etapa ( $1^{\text {a }}$ Etapa do Circuito Costa Doce - Seletiva para Seleção Gaúcha 2013, FGT). Os voluntários foram orientados quanto ao preenchimento do questionário e receberam informações a respeito da investigação. Todos os convidados que aceitaram participar, ou seu responsável legal quando menor de idade, assinaram o termo de consentimento livre e esclarecido.

$\mathrm{O}$ instrumento de registro dos dados foi completado logo após a pesagem e anteriormente ao início do campeonato. O preenchimento do mesmo foi realizado pelos próprios competidores, sob supervisão contínua e proximal de um dos autores, o qual esteve disponível para tirar dúvidas e oferecer esclarecimentos, sem interferir nas respostas emitidas. $\mathrm{O}$ instrumento usado foi um questionário sobre perda de peso em lutadores, o qual conta com validação prévia para a modalidade Judô. ${ }^{3}$ Foi necessária a adaptação do referido questionário, exclusivamente com respeito à modalidade Judô para TKD, uma vez que não existe um instrumento validado para este esporte. O instrumento é composto por três seções, a primeira 
referente a dados gerais dos participantes, a segunda destinada ao relato de informações sobre o histórico de peso e de dietas e a terceira versando sobre os métodos utilizados pelos atletas no processo de perda de peso nos últimos 12 meses.

Com esse instrumento foram avaliados diversos ítens, como categoria atual; se o lutador subiu de categoria; quanto pesava nas últimas férias; se já havia perdido peso para competir; maior quantidade de peso que já perdeu para competir e quantas vezes isso aconteceu no último ano; quantos quilos costuma perder antes das competições; quanto tempo antes da competição o atleta costuma aferir o seu peso; com que idade passou a perder peso para competir; quantos quilos costuma ganhar na semana posterior a uma competição; grau de influência exercida para a perda de peso. A partir da soma dos pontos acumulados a cada questão respondida, obteve-se escore Rapid Weight Loss Questionnaire (RWLQ), ${ }^{3}$ que indica a severidade do processo de perda de peso.

Os dados foram tabulados em planilha do Microsoft Excel $^{\circledR}$ e transportados para o software SPSS 17.0, onde foram analisados. Aplicou-se teste de normalidade (Shapiro-Wilk) que verificou distribuição assimétrica. Assim, empregaram-se mediana e semiamplitude interquartílica, respectivamente, como medidas de centralidade e dispersão. Para a comparação entre gêneros nas variáveis de interesse, foi aplicado teste de Mann-Whitney.
Adicionalmente, empregou-se uso de frequências absoluta e relativa e teste de qui-quadrado para testar diferentes proporções nas respostas emitidas. Assumiu-se 5\% como nível de significância estatística.

\section{RESULTADOS}

No momento do estudo, todos os participantes inscritos na competição eram faixa-preta e elegíveis, e não houve recusas ao envolvimento no estudo. Assim, foram incluídos 23 atletas, dos quais $15(65,2 \%)$ eram do sexo masculino e $14(60,8 \%)$ tinham menos de 18 anos. De todos os lutadores, apenas dois praticantes do sexo masculino relataram não perder peso para competir. Quanto ao nível competitivo, atletas do sexo feminino $(85,7 \%)$ e do sexo masculino $(92,8 \%)$ competiram em eventos regionais, obtendo medalhas. Percentuais semelhantes de participação ocorreram em campeonatos estaduais, respectivamente $85,7 \%$ e $73,3 \%$. No entanto, apenas $57,1 \%$ das mulheres e $40 \%$ dos homens obtiveram medalhas em eventos nacionais. A maioria dos lutadores $(71,4 \%$ das participantes do sexo feminino e $60 \%$ dos participantes do sexo masculino) não havia participado de competições internacionais, mas $28,5 \%$ das participantes do sexo feminino e $26,6 \%$ dos participantes do sexo masculino conquistaram medalhas. As demais características dos lutadores são apresentadas na Tabela 1.

Tabela 1. Característica de competidores de Taekwondo participantes da etapa seletiva para composição da Seleção Gaúcha do ano de 2013, da Federação de Taekwondo do estado do Rio Grande do Sul, segundo o sexo.

\begin{tabular}{|c|c|c|c|c|}
\hline \multirow{2}{*}{ Característica } & \multicolumn{2}{|c|}{ Sexo feminino $(n=8)$} & \multicolumn{2}{|c|}{ Sexo masculino $(n=15)$} \\
\hline & Mediana & $25 \%-75 \%$ & Mediana & $25 \%-75 \%$ \\
\hline Idade (anos) & 15 & $13-18$ & 17 & $13-23$ \\
\hline Início no Taekwondo (anos) & 7,5 & $5,5-10$ & 8 & $6-11$ \\
\hline Início em competições (anos) & 8,5 & $7-11$ & 10 & $7-13$ \\
\hline Massa corporal (kg) & 52,5 & $48,9-59$ & 61 & $48-75$ \\
\hline Estatura (m) & 1,59 & $1,57-1,65$ & 1,71 & $1,65-1,77$ \\
\hline Competições no último ano* & 1,5 & $0,5-3$ & 5 & $3-8$ \\
\hline Medalhas no último ano & 1,5 & $0,5-2,5$ & 3,5 & $2-8$ \\
\hline Massa corporal nas férias (kg) & 53 & $50-58$ & 68 & $43-75$ \\
\hline Maior perda de peso (kg) & 1,25 & $1-2$ & 1 & $1-2,5$ \\
\hline Vezes que perdeu peso - último ano & 1 & $0-1$ & 1 & $0-2$ \\
\hline Massa corporal perdida (kg) & 1 & $1-2$ & 1 & $0-3$ \\
\hline Ganho após pesagem (kg) & 2 & $1-7$ & 1,25 & $0-2$ \\
\hline Em quanto tempo perde (dias) & 3 & $3-3$ & 3 & $0-4$ \\
\hline Idade de início - perda de peso & 5 & $4-5$ & 4 & $0-5$ \\
\hline
\end{tabular}

* Diferença significativa entre sexos $(p=0,008)$. 
A Tabela 2 demonstra as pessoas que mais influenciaram as atitudes dos lutadores de TKD quanto às suas atitudes referentes à perda de peso. Destacase que médicos quase não influenciaram esse tipo de processo, e colegas de treino tiveram influência que vai de razoável a muita $(62,5 \%)$. A pessoa que mais influenciou foi o professor de TKD, com $77,79 \%$ dos relatos.

Na Figura 1 são apresentados os dados referentes aos métodos empregados para a perda de peso, segundo a frequência de utilização. Pode-se observar a omissão de refeições, aumento de exercícios físicos e treinar com agasalhos, plásticos ou roupas de borrachas como os usados mais comumente. O método mais empregado foi o aumento da quantidade de exercícios, seguido do treino com roupas de borracha e omissão de refeições. Métodos mais agressivos, como o uso de sauna, laxantes, diuréticos, inibidores de apetite, bem como provocação de vômito, foram substancialmente infrequentes.

Tabela 2. Grau de influência exercida por diferentes profissionais para a perda de peso em jovens lutadores de Taekwondo (com base nas respostas dos próprios atletas). Competidores de Taekwondo participantes da etapa seletiva para composição da Seleção Gaúcha do ano de 2013, da Federação de Taekwondo do estado do Rio Grande do Sul.

\begin{tabular}{|c|c|c|c|c|c|c|c|}
\hline \multirow{2}{*}{$\begin{array}{l}\text { Pessoa que exerceu } \\
\text { influência }\end{array}$} & \multicolumn{6}{|c|}{ Influência } & \multirow[b]{2}{*}{$\mathbf{p}$} \\
\hline & $\begin{array}{c}\text { Nenhuma } \\
\text { N (\%) }\end{array}$ & $\begin{array}{l}\text { Pouca } \\
\text { N (\%) }\end{array}$ & $\begin{array}{c}\text { Razoável } \\
\text { N (\%) }\end{array}$ & $\begin{array}{l}\text { Muita } \\
\text { N (\%) }\end{array}$ & $\begin{array}{l}\text { Não sei } \\
\text { N (\%) }\end{array}$ & $\begin{array}{l}\text { Total } \\
\text { N (\%) }\end{array}$ & \\
\hline Colega de treino & $5(31,25)$ & $1(6,25)$ & $5(31,25)$ & $5(31,25)$ & - & $16(100)$ & 0,39 \\
\hline Lutador mais velho & $3(17,66)$ & $5(29,41)$ & $4(23,52)$ & $5(29,41)$ & - & $17(100)$ & 0,88 \\
\hline Médico* & $13(81,25)$ & - & $1(6,25)$ & - & $2(12,5)$ & 16 (100) & 0,001 \\
\hline Preparador físico & $6(37,5)$ & - & $5(31,25)$ & $1(6,25)$ & $4(25)$ & $16(100)$ & 0,32 \\
\hline Mestre* & $3(16,6)$ & $1(5,55)$ & $4(22,24)$ & $10(55,55)$ & - & $18(100)$ & 0,02 \\
\hline Nutricionista & $5(29,43)$ & $4(23,52)$ & $1(5,88)$ & $7(41,17)$ & - & 17 (100) & 0,22 \\
\hline Outra pessoa* & $11(64,9)$ & $1(5,88)$ & $2(11,76)$ & $1(5,88)$ & $2(11,76)$ & 17 (100) & 0,001 \\
\hline
\end{tabular}

* Distribuições proporcionalmente desiguais.

Figura 1. Frequência de métodos utilizados para perda de peso rápida em jovens competidores de Taekwondo $(n=23)$. Competidores de Taekwondo participantes da etapa seletiva para composição da Seleção Gaúcha do ano de 2013, da Federação de Taekwondo do estado do Rio Grande do Sul.

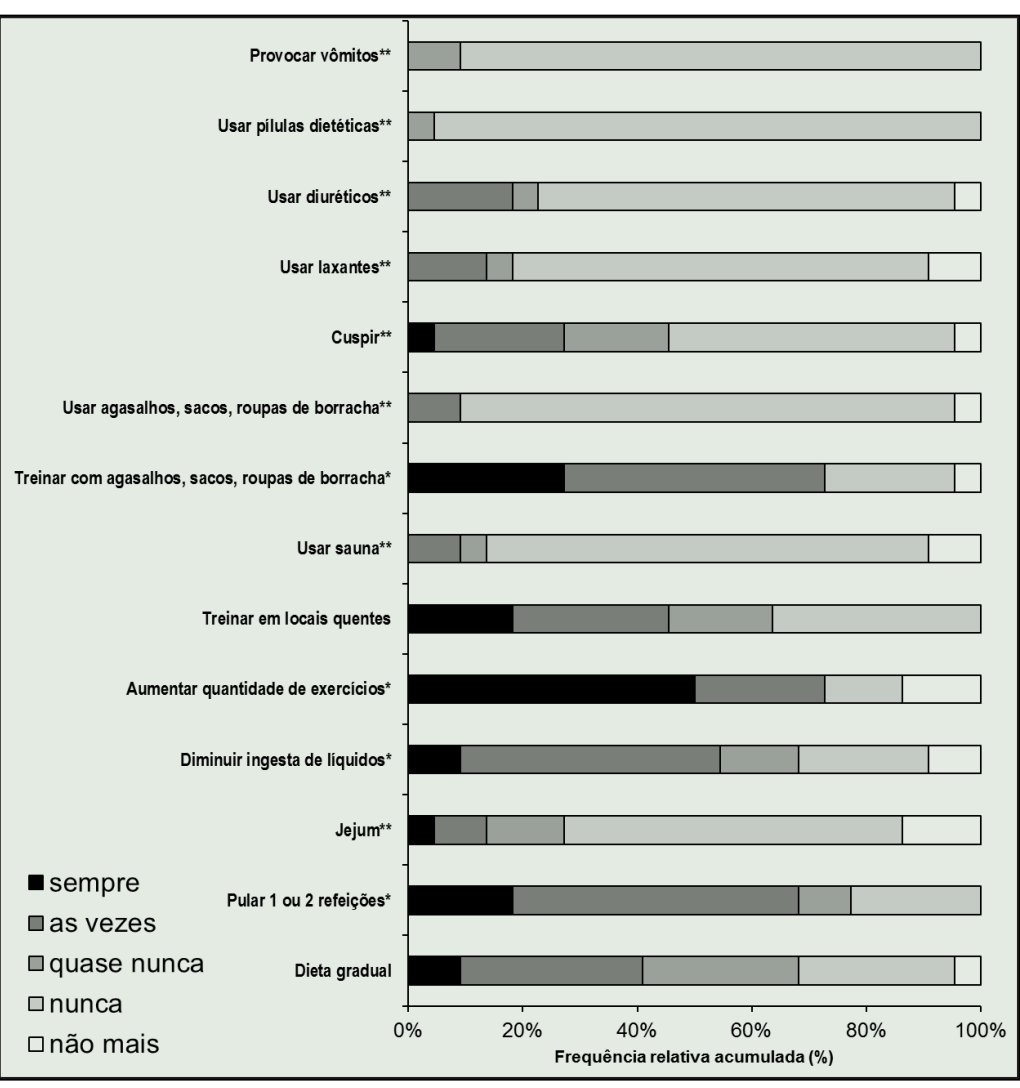

* $\chi^{2}$ com $\mathrm{p}<0,05 ;{ }^{* * *} \chi^{2}$ com $\mathrm{p}<0,001$, explicitando proporções desiguais entre métodos. 
Os participantes do sexo masculino exibiram escore de $25,3 \pm 15,8$ pontos no questionário padronizado sobre hábitos relacionados à perda de peso, ao passo que as participantes do sexo feminino apresentaram 25,5 $\pm 9,9$ pontos, sem diferenças significativas entre os sexos.

\section{DISCUSSÃO}

Este estudo, que objetivou estudar as características e práticas relacionadas à perda rápida de peso em lutadores gaúchos faixas-preta de TKD, identificou um alto percentual $(91,3 \%)$ de lutadores que perdem peso para competir. Destes, a maioria referiu diminuir de 1 a $3 \mathrm{~kg}$ no período anterior à competição, cerca de três a quatro dias até duas vezes por ano.

Estudo com praticantes de taekwondo da categoria juvenil, de ambos os sexos, que foram pesados aproximadamente 20 horas antes da luta e novamente minutos antes de entrarem em combate, apontou resultado parecido com o da presente investigação, em que $100 \%$ dos atletas revelaram perda e recuperação de, em média, 1,9 kg. ${ }^{8}$

Corroborando com os dados encontrados no presente estudo, relata-se que $87 \%$ dos atletas de TKD, de um clube de Londres, afirmaram perder peso no período antecedente à competição. ${ }^{6}$ Nessa pesquisa, 12 dos 30 lutadores referiram iniciar a prática de perda de peso duas semanas antes da luta, diferentemente do período de tempo de três a quatro dias encontrado no presente estudo. Esta diferença entre o período de tempo usado para perda de massa corporal pode ter ocorrido pelo fato de que os participantes do presente estudo terem perdido menos da metade do peso em relação aos atletas londrinos. ${ }^{6}$

Destaca-se ainda que a maior influência foi exercida pelo treinador, seguida pela influência dos colegas, dos pais e dos médicos. Neste contexto, verificou-se que os atletas não buscaram orientação com profissionais da área nutricional, e que os métodos usados foram sugeridos pelos treinadores e mestres. ${ }^{8}$ Partindo do princípio de que os lutadores perdem peso com objetivo de enfrentar adversários menores e mais fracos, ${ }^{3}$ acredita-se que o mestre é o primeiro a alertar e estimular o seu atleta para essa estratégia. Por outro lado, há evidência de que profissionais da área da saúde podem contribuir para uma redução mais saudável de peso corporal. ${ }^{9}$

Entre os londrinos, os métodos mais citados foram associação de restrição alimentar e hidrica com aumento de treinamento, aumento de atividade física ou apenas sauna, diferindo dos métodos evidenciados entre atletas gaúchos. ${ }^{6}$ Em lutadores de TKD canadenses registrou-se que o jejum foi o método mais frequente $(53 \%)$, e $83 \%$ dos voluntários referiram praticar exercício concomitantemente, o que também foi observado com atletas de Judô. ${ }^{5,10}$ Tais dados vão ao encontro dos obtidos com os participantes envolvidos no presente estudo, embora Artioli et al. ${ }^{11}$ tenham descrito que tanto as técnicas que promovem desidratação quanto os métodos de redução dietética severa podem provocar queda no desempenho aeróbio e anaeróbio. Possivelmente os lutadores fazem uso destes métodos por não reconhecerem tais prejuízos ou não perceberam seus efeitos deletérios sobre o organismo. ${ }^{11}$

Um estudo conduzido por Artioli et al..$^{10}$ revelou que atletas de Judô foram submetidos, em média, a três ciclos de perda de peso por temporada, e que essa prática teve início aos 12 anos de idade. Tais dados diferem dos achados nos atletas gaúchos de TKD, uma vez que se encontrou um único ciclo de perda de peso ao ano, porém, com início precoce, aos cinco anos de idade.

A redução do peso corpóreo no período anterior às competições é desejada porque algumas modalidades de luta apresentarem sua organização esportiva dividida em diferentes categorias baseadas na massa corporal. ${ }^{2}$ Então, com o objetivo de competirem com atletas menores e mais fracos, os lutadores preferem perder peso dias antes de enfrentarem seus adversários. ${ }^{4}$ Porém, como a maioria dos atletas busca competir com peso inferior ao seu peso habitual, muitos não conseguem mantê-lo e tornam a alcançá-los após a luta. ${ }^{12}$ Tais hábitos também foram encontrados entre os atletas gaúchos deste estudo, apesar de a rápida perda de peso ser técnica potencialmente perigosa, pois pode provocar efeitos negativos sobre os atletas, tais como aumento de tensão, tonturas, fadiga, aumento de confusão e perda de vigor. ${ }^{9}$

A flutuação de peso, conhecida como weight cycling, promove adaptações fisiológicas que resultam em maior eficiência para utilizar e armazenar energia. ${ }^{12}$ Complementarmente, existe evidência de que a perda de peso rápida está associada à desidratação intencional, principalmente em algumas modalidades esportivas de combate. Recentemente, um estudo demonstrou que a exposição ao calor e/ou a desidratação podem prejudicar o desempenho físico. ${ }^{13}$ Isto se dá em função de o estado de desidratação causar diminuição no volume de ejeção e no débito cardíaco, reduzindo o volume plasmático e, consequentemente, limitando a oxigenação dos músculos durante o exercício. ${ }^{12}$ No entanto, estudo recente apontou como sendo provável que a perda hídrica não comprometa a saúde e não 
diminua o desempenho físico de atletas de modalidades esportivas de combate. ${ }^{14}$

Apesar da verificação de weight cycling neste estudo e na maioria dos estudos citados neste artigo, parece que essa estratégia, apesar de muito usada, não tem relação com o bom desempenho nas competições de TKD. Kazemi et al. ${ }^{5}$ concluíram que não existe relação entre recuperação da massa corporal e ganho de medalhas, ou seja, entre 108 atletas estudados em competição de juniores (15 a 17 anos), o número de campeões não esteve associado a quantidade de atletas que mais recuperaram peso antes da luta, fato responsável pelo aumento do desempenho.

Ao se considerar o escore do RWLQ ${ }^{3}$ referente à perda de peso, os sujeitos do presente estudo exibiram aproximadamente 25 pontos, sem diferenças estatísticas entre sexos, o que corrobora com achados prévios. ${ }^{10}$ Apesar de escassos, escores de outros grupos de lutadores tendem a ser superiores. Com lutadores de Judô, observou-se progressividade de acordo com o nível competitivo (regional 17,5 pontos, estadual 25 pontos, semelhante ao deste estudo, nacional 28 pontos e internacional $\geq 30$ pontos $)^{10}$. Ao se considerar a faixa etária, exibe-se tendência linear de diminuição: crianças de 9-11 anos atingem 35,8 pontos, ao passo que adultos com mais de 20 anos chegam a 22,9 pontos. ${ }^{10}$ Em amostra com 94 lutadores de Judô para validação do respectivo instrumento, registrou-se escore de 24,9 pontos no teste e 24,1 pontos no reteste, valores inferiores aos obtidos com lutadores de TKD. ${ }^{3}$

Contudo, o presente estudo apresenta algumas limitações no que diz respeito ao método, sendo o emprego de questionário não validado para o tipo de luta específico uma delas. Apesar de que o mesmo tem sido usado em diferentes investigações e responde aos objetivos da pesquisa ${ }^{1,3,5,10-12}$, o questionário aplicado foi validado apenas para atletas do Judô. Deve-se considerar que não existe questionário específico para o TKD, e as variáveis avaliadas no referido instrumento englobam os aspectos mais importantes para a pesquisa. $\mathrm{O}$ fato dos dados terem sido coletados durante apenas um evento esportivo de TKD poderia ser considerado cmo outra limitação deste estudo, entretanto, este é um dos eventos competitivos mais importantes do Rio Grande do Sul, e envolve lutadores de diferentes regiões do estado. Mesmo assim, novos estudos deverão considerar maior quantidade de eventos e de atletas, envolvendo competidores com graduações inferiores à faixa preta.

Considerando que o TKD é uma modalidade olímpica de combate, que existem competições tanto em nível estadual quanto mundial, e que a rápida redução de peso pode provocar queda no desempenho e diferentes prejuízos ao longo da carreira, ${ }^{12}$ os métodos de treinamento e as práticas de manutenção de peso devem ser ponderadas, uma vez que a rápida perda de peso pode prejudicar a saúde. ${ }^{12}$

Com vista à diminuição desta prática, medidas como pesagem dos atletas e exame do estado de hidratação imediatamente antes do início das lutas e criação de programas para educar e conscientizar a respeito dos problemas relacionados à rápida perda de peso, podem ser adotadas pelas confederações, federações e demais entidades reguladoras do esporte..$^{12}$ Estratégias semelhantes já foram adotadas com praticantes de luta olímpica nos Estados Unidos, as quais apresentaram resultados positivos. ${ }^{15}$

Em conclusão, o presente estudo observou que a perda rápida de peso é frequente entre lutadores de TKD, não há diferenças entre atletas femininos e masculinos e os métodos mais utilizados foram aumento de atividade física, omissão de refeições e restrição da ingestão hídrica. Por fim, destaca-se que a pessoa que mais estimula e orienta esta prática é o próprio mestre/professor da modalidade, seguido de companheiros de treino.

\section{REFERÊNCIAS}

1. Fabrini SP, Brito CJ, Mendes EL, Sabarense CM, Marins JCB, Franchini E. Práticas de redução de massa corporal em judocas nos períodos pré-competitivos. Rev Bras Educ Fís Esporte. 2010;24(2):165-77.

2. Rossi L, Tirapegui J, Castro IAD. Restrição moderada de energia e dieta hiperprotéica promovem redução ponderal em atletas de elite do Karatê. Rev Bras Ci Mov. 2004;12(2):69-73.

3. Artioli G, Scagliusi F, Kashiwagura D, Franchini E, Gualano B, Junior AL. Development, validity and reliability of a questionnaire designed to evaluate rapid weight loss patterns in Judo players. Scand J Med Sci Sports. 2009;20(1):177-87.

4. Chagas CEA, Ribeiro SML. Avaliação de uma intervenção nutricional convencional em atletas de luta. Braz J Spor Nutr. 2012;1(1):1-9.

5. Kazemi M, Shearer H, Choung YS. Pre-competition habits and injuries in Taekwondo athletes. BMC Musc Disor. 2005;6(26):1-9.

6. Fleming S, Costarelli V. Eating behaviours and general practices used by Taekwondo players in order to make weight before competition. Nutr Food Sci. 2009;39(1):16-23. 
7. Franchini E, Del Vecchio FB. Estudos em modalidades esportivas de combate: estado da arte. Rev Bras Educ Fís Esporte. 2011;25(nesp): 67-81.

8. Kazemi M, Rahman A, Ciantis MD. Weight cycling in adolescent Taekwondo athletes. J Can Chiropr Assoc. 2011;55(4):318-24. doi: 0008-3194/2011/318-324/\$2.00/CJCCA 2011.

9. Morton JP, Robertson C, Sutton L; Maclaren DP. Making weight: a case study from professor boxing. Int J Sports Nutr Exerc Meta. 2010;20(1):80-5.

10. Artioli GG, Gualano B, Franchini E, Scaglius FB, Takesian M, Fuchs M, Lancha AH Jr. Prevalence, magnitude and methods of rapid weight loss among judo competitors. Med \& Sci Sports Exerc. 2010;42(3):436-42.

11. Artioli GG, Scagliusi FB, Polacow VO, Gualano B, Lancha Junior AH. Magnitude e métodos de perda rápida de peso em judocas de elite. Rev Nutr. 2007;20(3):307-15.

12. Artioli GG, Franchini E, Lancha Junior AH. Perda de peso em esportes de combate de domínio: revisão e recomendações aplicadas. Rev Bras Cineantropom Desempenho Hum. 2006;8(2):92-101.

13. Kraft JA, Green JM, Bishop PA, Richardson MT, Neggers YH, Leeper JD. The influence of hydration on anaerobic performance: a review. Res Quar Exerc Sport. 2012;83(2):282-92.

14. Langan-Evans C, Close GL, Morton JP. Making weight in combat sports. J Strength Cond. 2011;33(6):26-39.

15. Davis SE, Gregory BD, Kristy R, Christopher B, Jelena S, Melissa S. Preliminary Investigation: The impact of the NCAA wrestling weight certification program on weight cutting: J Strength Cond. 2002;16(2):305-7. 